\title{
Editorial to the Special Issue: Modeling and Characterization of Low Permeability (Tight) and Nanoporous Reservoirs
}

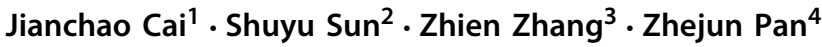

Published online: 4 January 2019

(c) Springer Nature B.V. 2019

In recent years, low permeability (tight) and nanoporous reservoirs have attracted much attentions for their special properties. Compared to conventional reservoirs, these unconventional oil and gas reservoirs possess low permeability (tight) and have nanoscale pores where oil and gas are trapped, indicating distinct multiscale and heterogeneous characteristics. Its complex microstructures and non-Darcy properties make the traditional methods and modeling technologies used in conventional reservoirs not feasible to be effectively applied in unconventional oil and gas reservoirs. Therefore, novel methods are required to be developed to model and characterize low permeability (tight) and nanoporous reservoirs and to describe the flow and transport mechanisms of multiscale and multiphysics nature.

Since unconventional reservoirs are a complex multiscale system, the production of oil and gas requires underground fluids passing through different scales of seepage channels such as micro-nano pores, natural micro-macro fractures, and multi-level hydraulic fractures, which is a complex process across time and space scales. Moreover, there are physical and chemical interactions between fluid and rock under thermal and mechanical conditions. Therefore, the effective development of unconventional oil and gas resources is facing enormous challenges. The focus of this special issue in Transport in Porous Media aims to present recent advances in various subjects addressing new multiscale and multiphysics approaches and technologies in the unconventional resources.

Twelve papers are included in this special issue, including many aspects in modeling and characterization of the tight and nanoporous reservoirs. The paper "A feature-based stochastic permeability of shale" by Singh and Cai includes two parts. The Part 1 -validation and two-

Zhien Zhang

zhang.4528@osu.edu

Jianchao Cai

caijc@cug.edu.cn

Shuyu Sun

shuyu.sun@kaust.edu.sa

Zhejun Pan

zhejun.pan@csiro.au

1 China University of Geosciences, Wuhan, People's Republic of China

2 King Abdullah University of Science and Technology, Thuwal, Kingdom of Saudi Arabia

3 Ohio State University, Columbus, USA

4 CSIRO, Clayton, VIC, Australia 
phase permeability in a Utica shale sample proposes a novel stochastic method to predict the permeability of a fractured shale system by discretizing shale into matrix and fractures and the new analytical expressions of permeability to account for non-ideal nature of porous medium and two-phase flow in fractures. The Part 2-predicting field-scale permeability applies the proposed method to predict field-scale permeability through an optimization process that utilizes field-scale production and other readily available information.

The paper "Fractal and multifractal characteristics of pore throats in the Bakken shale" by Liu et al. uses a high-pressure mercury intrusion method to analyze the information of a few samples from the Bakken Shales. Information regarding the pores of shales such as porethroat size, pore-throat ratio, and fractal and multifractal behaviors is derived and compared.

Spontaneous imbibition of silicone oils is reported in the paper titled "Capillarity-driven oil flow in nanopores: Darcy scale analysis of Lucas-Washburn imbibition dynamics" by Gruener et al. Silicone has a slightly more complex molecules structure, in mesoporous, monolithic silica glass with pore diameters of 6.5 and $10 \mathrm{~nm}$. The authors also perform gravimetrical experiments and analyze the scaling of the measured imbibition kinetics based on a Darcy effective medium ansatz.

The paper "Modelling imbibition processes in heterogeneous porous media" by Suo et al. develops a comprehensive numerical framework to solve imbibition problems in heterogeneous porous media based on the nonlinear Richard's equation with consideration of spatial distribution of properties. Through special interface treatments, this model is extended to more complex domains. The validation of the current model with existing experimental results and the simulations on a series of two-dimensional cases are also performed.

In the paper "Pore network modeling of shale gas reservoirs: Gas desorption and slip flow effects," the authors Foroozesh et al. investigate the contribution of slip and desorption effects in terms of velocity enhancement. A dynamic pore-network flow model is created to allow for a more accurate quantification of slip and desorption effects on shale gas flow.

The flow mechanism and simulation approaches of shale gas reservoirs are reviewed in the paper titled "Flow mechanism and simulation approaches for shale gas reservoirs: A review" by Zhang et al. First, the adsorption and desorption process is introduced, as well as the flow regime description. Besides, numerical simulation on micro- and mesoscales, e.g., molecular dynamics and lattice Boltzmann method, are reviewed. Meanwhile, the apparent permeability correction, which is the macroscopic focus, is concluded. The gas flow simulations at reservoir scales, including numerical models and the effect of geomechanics, are discussed.

The paper "A collection of analytical solutions for the flash equilibrium calculation problem" by Mikyška aims to develop a collection of suitable problems with known analytical (or closed-form) solutions, which can be used as benchmark solutions when testing convergence of the numerical solutions computed by flash equilibrium calculation codes. To obtain a problem possessing an analytical solution, the author considers a special form of the free energy. The procedure is illustrated and further generalized based on special cases.

In the paper "Estimation of sandstone permeability with SEM images based on fractal theory," Yu et al. presents a relationship between micropore fractal characteristics obtained from SEM images and macroscopic permeability based on the method of microfractal characteristics. In addition, the authors propose a reliable method for calculating rock and soil permeability using SEM images, as well as a calculation method of permeability based on fractal parameters obtained from image processing.

The paper "Reduced-order modeling of subsurface multi-phase flow models using deep residual recurrent neural networks" by Kani et al. presents a reduced-order modeling technique for subsurface multiphase flow problems building on the deep residual recurrent neural network (DR-RNN). The authors combine DR-RNN with proper orthogonal decomposition 
and discrete empirical interpolation method to reduce the computational complexity associated with high-fidelity numerical simulations. Finally, the proposed reduced model is also demonstrated.

Al-Rbeawi introduces the inflow performance relationships of unconventional reservoirs considering multiphase flow conditions in the porous media in the paper titled "Transient and pseudo-steady-state inflow performance relationships for multiphase flow in fractured unconventional reservoirs". Early production time transient state flow IPR and late production time pseudo-state IPR are considered for constant sandface flow rate and constant wellbore pressure. Several deterministic models for reservoir total mobility and compressibility are generated from PVT data and relative permeability curves. The analytical models of the flow regimes, developed in bounded hydraulically fractured unconventional reservoirs, are included the impact of multiphase flow condition and used to generate transient and pseudosteady-state IPRs.

The last paper "Numerical modeling and simulation of shale-gas transport with geomechanical effect" by El-Amin et al. is devoted to employing a mixed finite element method to simulate the problem of shale gas transport using the dual-porosity, dual-permeability model including the rock stress-sensitivity. The authors provide a numerical analysis on the stability of the mixed finite element method for this system and establish a mathematical foundation, in addition to selective numerical tests.

The papers in this special issue provide a diverse overview of multiscale and multiphysics approaches and technologies used in unconventional resources, which cover several aspects of low permeability (tight) and nanoporous reservoirs, such as the new method to predict the reservoir properties, pore scale modeling of multiphase flow, and nanoscale effect of shale gas. However, more innovative and in-depth investigations on the unconventional resources still require future research.

Publisher's Note Springer Nature remains neutral with regard to jurisdictional claims in published maps and institutional affiliations. 